\title{
Metal ductility evaluation by flattening test: The geometry dependence
}

\author{
Yao Wang ${ }^{\mathrm{a}}$, Guangxue Yang ${ }^{\mathrm{a}}$, Xianqi Lei ${ }^{\mathrm{b}}$, Qiang $\mathrm{Li}^{\mathrm{a}}$, Lijian Rong ${ }^{\mathrm{c}}$, Xiaofeng $\mathrm{Hu}^{\mathrm{c}}$, Yujie Wei ${ }^{\mathrm{a}, \mathrm{b}, *}$ \\ ${ }^{\text {a }}$ Engineering Research Center of Structure Reliability and Operation Measurement Technology of Rail Guided Vehicles, Ministry of Education, Beijing Jiaotong University, \\ Beijing, 100044, China \\ ${ }^{\mathrm{b}}$ State Key Laboratory of Nonlinear Mechanics (LNM), Institute of Mechanics, Chinese Academy of Sciences, Beijing, 100190, China \\ ${ }^{\mathrm{c}}$ Key Laboratory of Nuclear Materials and Safety Assessment, Institute of Metal Research, Chinese Academy of Sciences, Shenyang, 110016, China
}

\section{A R T I C L E I N F O}

\section{Keywords:}

Flattening test

Plastic deformability

Gurson-Tvergaard-Needleman model

Failure

Finite element analysis

\begin{abstract}
A B S T R A C T
The reliability of pipes, tubes and liquid tanks (cylinders) is of paramount significance to our life. The typical way to examine the ability of those structures to undergo plastic deformation is to apply a flattening test. In this paper, we used the Gurson-Tvergaard-Needleman damage model and finite element simulations to capture the flattening of a tube made of typical pipe materials. We demonstrated how the tube thickness, radius, and length would affect the critical displacement where tube failure initiates. For flattening with platens, the failure displacement first increases with tube length and reaches a peak value, and it then decreases and converges to a constant value for a particular geometry. The failure initiates at the two edges of short tubes, but shifts to the center in intermediate tubes where the failure displacement maximizes. Failure then always starts from the middle in even longer tubes. In contrast, flattening with indenters exhibits two peaks in the compressible displacement vs. tube length curve. In the end, we proposed effective experimental strategies to obtain the intrinsic ability of tubes under plastic deformation. The results reported here could be employed to characterize the mechanical properties of materials for pipes, tubes and tanks, and they could also be applied to guide the engineering design of such structures.
\end{abstract}

\section{Introduction}

Pipes, tubes and liquid tanks (cylinders) are commonly seen facilities in engineering practice. Their reliability is of paramount significance to our daily life. In addition to normal tensile, compression, fracture, and fatigue properties of the materials that the structures are made of, the mechanical behavior of tubes as a structure is also essential for their reliability assessment. The elastic instability of typical tubes, in particular circular tubes, has been investigated intensively. Theoretical analysis on the plastic deformability of tubes has been rarely seen. Experimentally, a typical technique employed in the field is the so-called flattening test. Being one type of the ductility testing, flattening radically a tube or a test piece from pipelines or tanks or gas cylinders is able to examine the capability of plastic deformation as well as the initial quality of the structures, which has been broadly employed in the field [1-7]. So far, a subcommittee of the International Organization for Standardization specifies some geometrical parameters for testing pieces [8]. However, there lacks systematic investigation on the detailed geometry specifications for valid information from flattening tests. In engineering practice and for economic consideration, there may be circumstances we are not able to fulfill all requirements of the testing conditions. In addition, failure modes and failure patterns may differ from one working piece to another. All those considerations call for a systematic investigation on the applicability of the flattening tests to particular engineering problems. In this manuscript, we supplied a comprehensive numerical investigation on the deformation and failure process of flattening typical pipe steels with different geometries.

\section{Numerical modelling}

The failure in X80 tube steel is similar to typical ductile fracture of many other metals. In a flattening test, part of the tube is under severe bending. Damage determined by the combined effect of stress triaxiality and equivalent strain initiates and grows to form macroscopically perceivable cracks. There exist many constitutive models which are capable of capturing the whole deformation process involving plasticity, void nucleation, void growth, coalescence, and macroscopic fracture till material failure (e.g. Refs. [9-17]). For convenience, we adopted the Gurson-Tvergaard-Needleman (GTN) model [9-13] embedded in ABAQUS [18] to capture the plastic deformation and damage evolution of the material. The plastic potential in the GTN model [18] is given as

\footnotetext{
${ }^{*}$ Corresponding author.State Key Laboratory of Nonlinear Mechanics (LNM), Institute of Mechanics, Chinese Academy of Sciences, Beijing, 100190, China.

E-mail address: yujie_wei@lnm.imech.ac.cn (Y. Wei).
} 
$\Phi=\left(\frac{\sigma_{e}}{\sigma_{0}}\right)^{2}+2 q_{1} f^{*} \cosh \left(\frac{3 q_{2} \sigma_{m}}{2 \sigma_{0}}\right)-\left(1+q_{3} f^{* 2}\right)$

where $\sigma_{e}$ and $\sigma_{m}$ are the effective (von Mises) stress and the hydrostatic tension at a material point, $\sigma_{0}$ is the initial yield strength of the material, $q_{1}, q_{2}$ and $q_{3}$ are the material-dependent correction parameters, and $f^{*}$ is a function of void volume fraction $f$ :

$f^{*}=\left\{\begin{array}{cl}f & , \text { if } f \leq f_{c} \\ f_{c}+\frac{1}{q_{3}} \frac{q_{1}+\sqrt{q_{1}^{2}-q_{3}}-q_{3} f_{c}}{f_{F}-f_{c}}\left(f-f_{c}\right) & , \text { if } f_{c}<f<f_{F} \\ \frac{q_{1}+\sqrt{q_{1}^{2}-q_{3}}}{q_{3}} & , \text { if } f \geq f_{F}\end{array}\right.$

where $f_{c}$ is a critical value of the void volume fraction, and $f_{F}$ is the value of void volume fraction when the material has no load-carrying capacity. In the GTN model, the void volume fraction $f$ starts from an initial volume fraction $f_{0}$ and evolves in the following manner:

$\dot{f}=(1-f) \dot{\boldsymbol{\varepsilon}}^{p l}: \mathbf{I}+\frac{\dot{\bar{\varepsilon}}^{p l} f_{N}}{\sqrt{2 \pi} S_{N}} \exp \left[-\frac{1}{2}\left(\frac{\bar{\varepsilon}^{p l}-\varepsilon_{N}}{s_{N}}\right)^{2}\right]$

Where $\dot{\boldsymbol{\varepsilon}}^{p l}$ is the of plastic strain rate tensor and $\mathbf{I}$ is the unit tensor, and $\dot{\bar{\varepsilon}}^{p l}$ is the equivalent plastic strain rate, $\dot{\bar{\varepsilon}}^{p l}=\sqrt{2 / 3\left[\operatorname{dev}\left(\dot{\boldsymbol{\varepsilon}}^{p l}\right): \operatorname{dev}\left(\dot{\boldsymbol{\varepsilon}}^{p l}\right)\right]}$, and $\bar{\varepsilon}^{p l}=\int_{0}^{t} \dot{\bar{\varepsilon}}^{p l} d t$. Here $f_{N}, \varepsilon_{N}$ and $s_{N}$ are material parameters associated with void nucleation rate. All parameters used in our simulations are given in Table 1 .

In order to obtain accurate deformation process during the flattening test for X80 tube steel, we first calibrated the material model used for finite element simulations. We examined the stress-strain behavior of the material under uniaxial tension. The sample layout and geometries are given in Fig. 1a. The tested stress-strain curve of the material is shown in Fig. 1b. From the experimental measurement, we had the initial yield strength of the X80 steel of $\sigma_{y}=551 \mathrm{MPa}$. Because the way to determine the parameters has no generally accepted recommendations and these parameters are only material dependent, we used the so-called inverse finite element method and the recommended values from the ABAQUS documents to determine these parameters of GTN model [18-20]. By using the parameters tabulated in Table 1, we are able to capture the tensile stress-strain curve of the material seen in Fig. 1b, as well as the failure point. The selection of these parameters takes into account the perfect production of the tube (or pipe) without any defects. The deformation process of the dog-bone sample from our finite-element simulations is shown in Fig. 1c, which, in turn, shows the initial meshed three-dimensional sample, the necking process, and the final fractured sample. Both stress-strain curves and the deformation patterns match well with experimental observations.

The calibrated parameters were then used to model the flattening tests. We numerically performed the flattening tests by placing the testing piece between two platens and then flattened the piece by moving the platens in the direction perpendicular to the longitudinal axis of the tube. Typical tools used for flattening could be either platens or indenters, and here we modeled both cases to see the difference. To be consistent with experiments, we used a friction coefficient of $\mu=0.3$ between the flattening tools (platen or indenter) and the tube sample. For both types of flattening tools, the lower part was fully constrained, and the upper part only had the translational degree of freedom along the flattening direction. No constraints were imposed to the two ends of

Table 1

Material parameters used the GTN model to match the stress-strain curve of X80 under tension.

\begin{tabular}{lllllllll}
\hline$q_{1}$ & $q_{2}$ & $q_{3}$ & $f_{0}$ & $\varepsilon_{N}$ & $s_{N}$ & $f_{N}$ & $f_{c}$ & $f_{F}$ \\
\hline 1.25 & 1 & 1.5625 & 0 & 0.3 & 0.01 & 0.04 & 0.045 & 0.065 \\
\hline
\end{tabular}

all tubes except in plane-stress or plane-strain deformation.

According to the ISO standard [8], the width of platens should be at least 1.6 times of the outer diameter of the tube $(400 \mathrm{~mm})$, while the length of the platens should be longer than the sample(the maximum length: $2000 \mathrm{~mm}$ ). So we determined the dimensions of the platens: the width is $640 \mathrm{~mm}$, and the length is $2100 \mathrm{~mm}$. The section dimensions of the indenters are shown in Fig. 9a. Based on the same method to the platens, the width of the indenters should be taken $640 \mathrm{~mm}$. Both the platens and the indenters are analytical rigid bodies during the whole process of simulations.

In order to sum up the reliable mechanical laws from the comparison of the tube simulation results, we fully considered the influence of element size on simulation results. The element sizes of each case are actually the same.

We first show in Fig. 2 the flattening test results from FEM simulations where the load is applied by pushing two platens, with the initial and final geometries of the tube shown in Fig. 2a. The load-displacement curves from flattening tubes of different thicknesses from our finite-element simulation are given in Fig. 2b. A side view of the setup (viewing along the tube axis) is shown as the inset in Fig. 2b. In response to flattening, the load-displacement curves of tubes have a linear elastic region, where the deformation is elastically stable. Further compression may lead to plastic deformation in either top or bottom surfaces of the tube. The load then increases slowly but with a progressively increasing slope. At a critical displacement, the plastic deformation in the two ends of the compressed tube excesses its limitation for fracture and macroscopic failure occurs. A sudden load drop accompanies the macroscopic failure, as seen in Fig. $2 \mathrm{~b}$.

\section{Results on flattening test by platens}

In Fig. 3, we show the dependence of load and displacement at the failure point as a function of tube thickness. For a tube of the constant outer radius of $200 \mathrm{~mm}$, increasing tube thickness leads to the drop of compressibility, as seen in Fig. 3a. The load at the failure point, due to the increasing tube thickness, increases with tube thickness, as shown in Fig. 3b. The detailed deformed patterns of the cross-section of the tube are shown in Fig. 4. Here we examined the influence of tube length on deformation patterns. Fig. $4 \mathrm{a}-\mathrm{c}$ shows the evolution of patterns at different snapshots from the flattening simulation of tubes of the same outer radius of $200 \mathrm{~mm}$ and thickness of $20 \mathrm{~mm}$ but varying length, ranging from an ultra-short tube (plane stress deformation along the tube axis), a tube of finite length (length of $80 \mathrm{~mm}$ ), and an infinite long tube (plane strain deformation along the tube axis), respectively. The corresponding load-displacement curves are shown in Fig. 4d. In correlation with the curves, the initially circular cross-section (at 'a') becomes elastically instable at ' $b$ '. From ' $b$ ' to 'f', the span between the two contact lines formed by the platens and the tube becomes wider. At ' $\mathrm{g}$ ', the two ends of the compressed tube are under excessive plastic deformation, which causes macroscopic failure of the tube and leads to load drop.

In Fig. 5, we further explore the influence of tube length on the critical compressible displacement and corresponding critical load when failure initiates. For the same tube outer radius and tube thickness simulated in Fig. 4, we see that when the length of the tube is less than $15 \%$ of the tube outer radius, increasing tube length leads to a rapid increase in failure displacement, as seen in Fig. 5a. The peak value occurs when the tube length is about $30 \mathrm{~mm}$ (i.e. $15 \%$ of the tube outer radius). Then it decreases with further increase in tube length, and converges slowly to the value corresponding to the infinitely long tube case (plane strain condition in the tube axis direction). The failure load per tube length versus tube length curve shown in Fig. 5b exhibits a similar trend. Fig. $5 \mathrm{c}-\mathrm{f}$ shows the damage contours in tubes of different lengths at their respective failure displacement. The initial increasing failure compressible displacement as tube length increases is correlated with the transitional failure mode: There is concentrated damage 


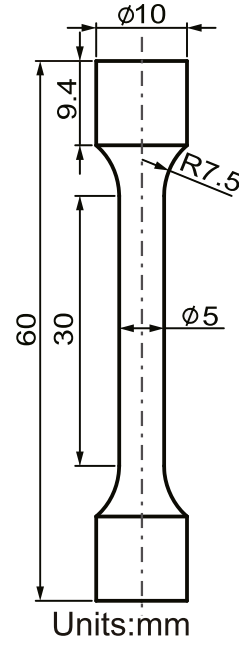

(a)

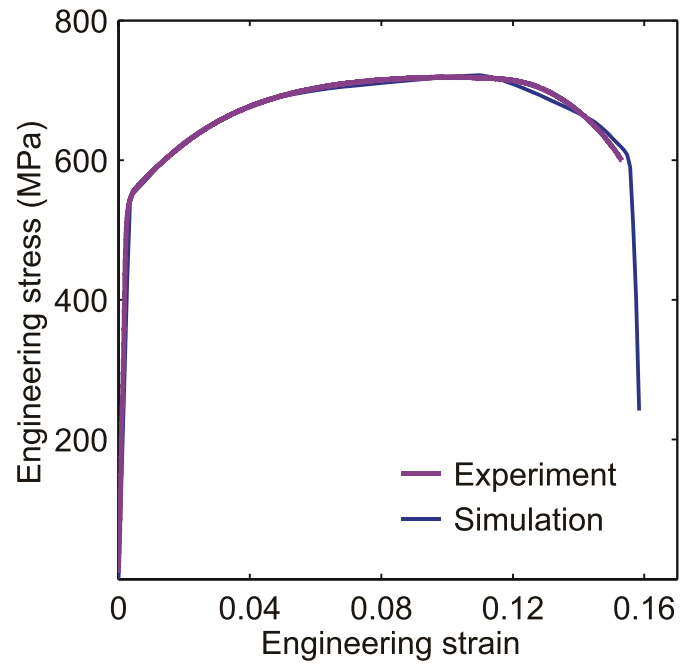

(b)

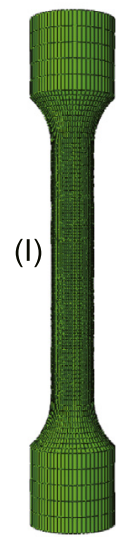

(c)

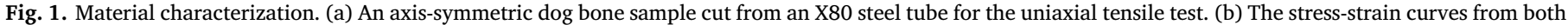

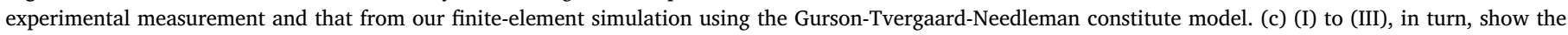
initial meshed sample, the necking, and final fracture sample.
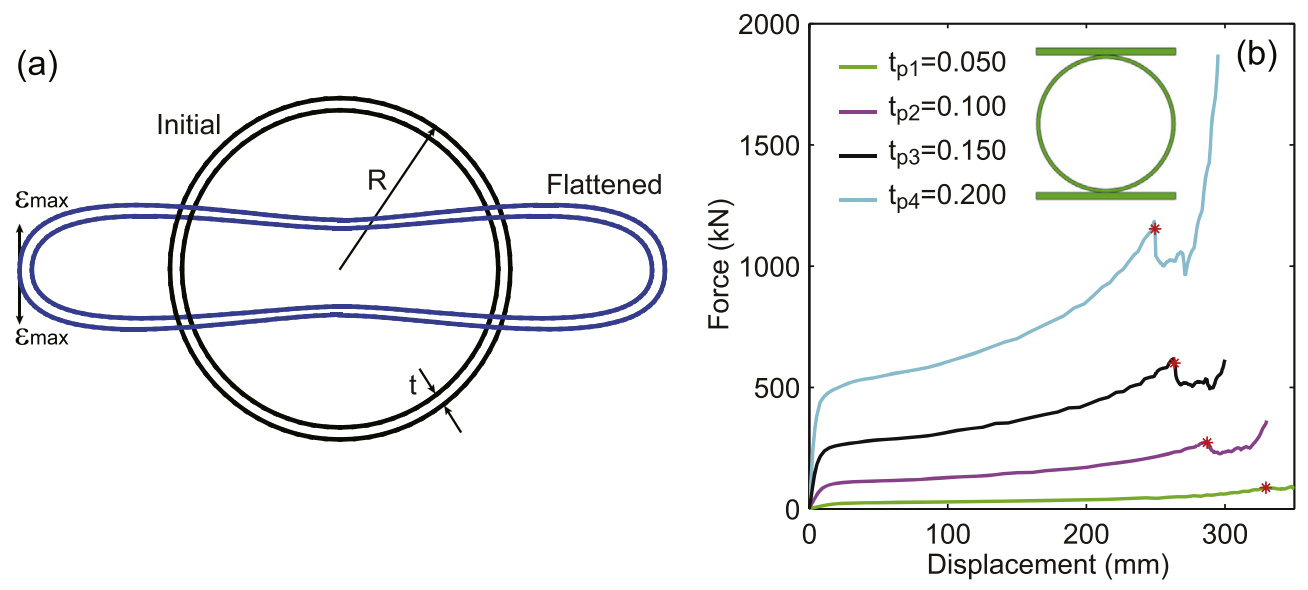

Fig. 2. Flattening simulations with platens. (a) Illustration to show the initial and the flattened tube. Typically failure initiates at the two ends where local tensile strain maximizes. (b) The load-displacement curves from flattening tubes of different thicknesses $\left(t_{p}\right.$ normalized by outer radius $R$ ). The inset shows the setup for finite-element simulations.
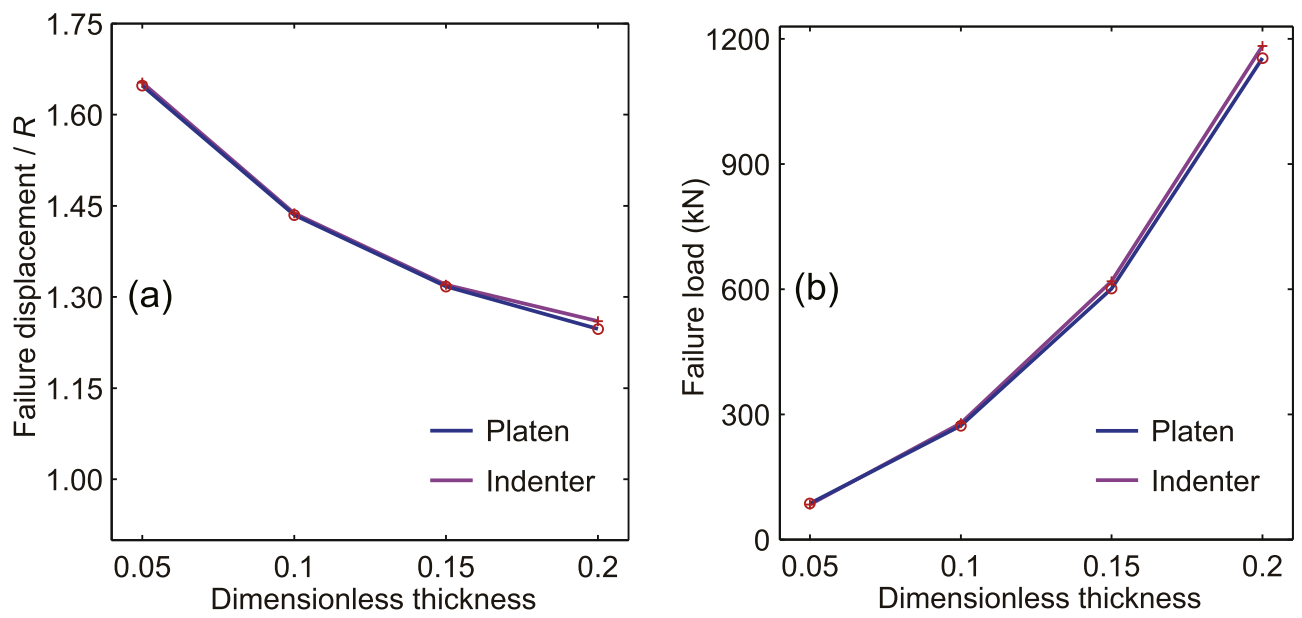

Fig. 3. The displacement (divided by tube outer radius $R$ ) and load at the failure point as a function of tube thickness (normalized by $R$ ). A constant tube outer radius of $R=200 \mathrm{~mm}$ is used. (a) The dependence of failure displacement as a function of tube thickness. (b) Failure load (corresponding to the failure displacement) as a function of tube thickness. 

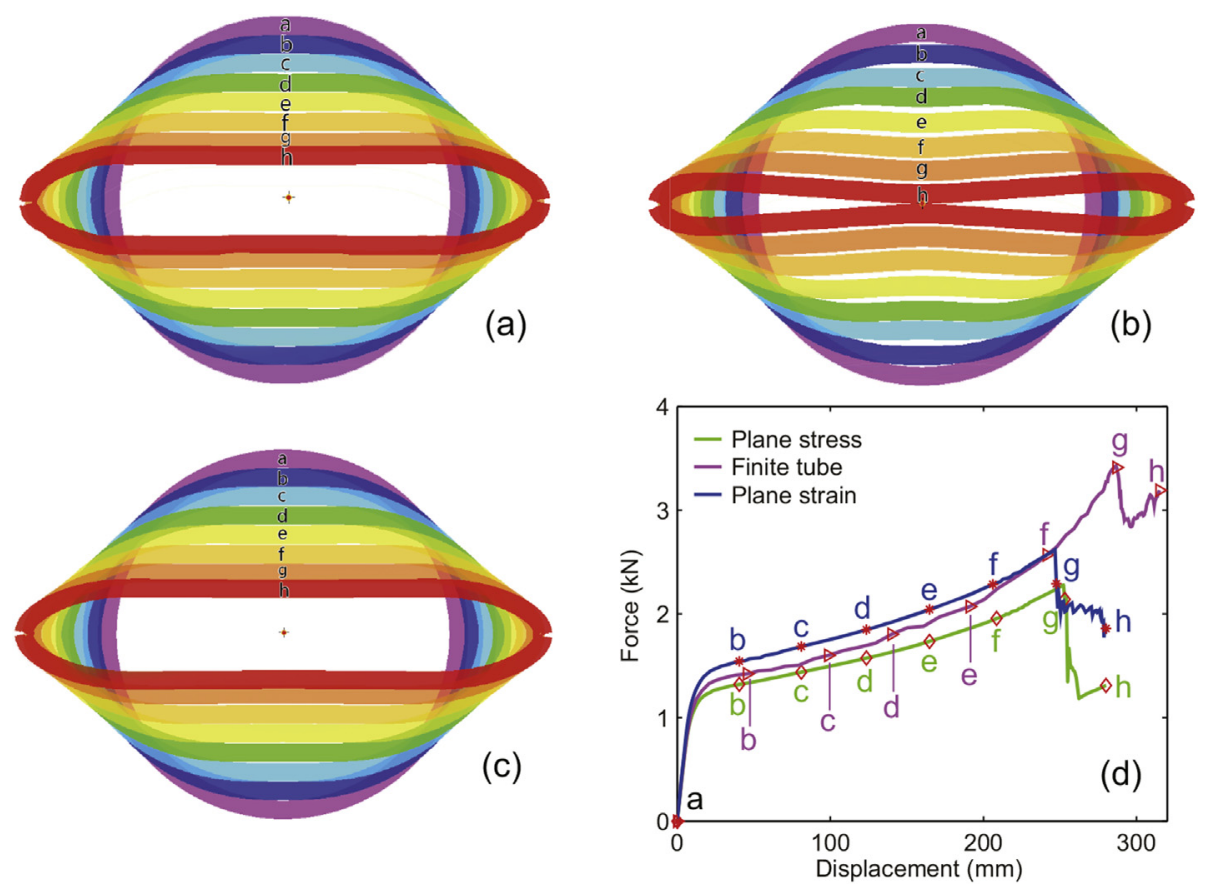

Fig. 4. The cross-section of deformed tubes of the same thickness (tube outer radius $R=200 \mathrm{~mm}$, thickness $20 \mathrm{~mm}$ ) but different length. The patterns at different snapshots corresponding to displacement keyed in (d). (a) Plane stress condition (ultra short tube). (b) A finite tube of length $80 \mathrm{~mm}$. (c) Plane strain condition (infinite long tube). (d) The respective load-displacement curves for the three cases.
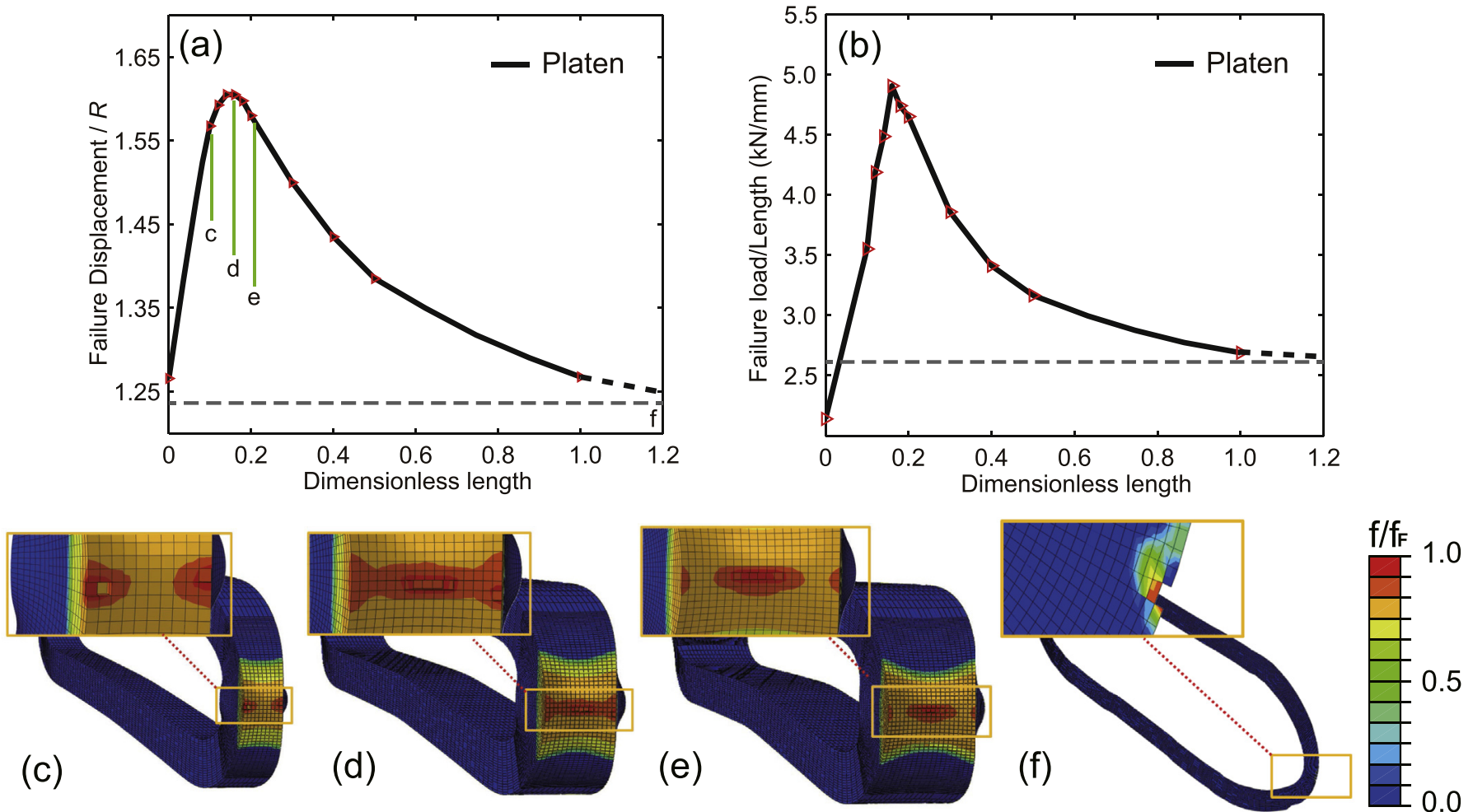

Fig. 5. The mechanical behavior in tubes of different length (normalized by tube outer radius $R$ ) but of the same thickness. (a) The variation of failure displacement (divided by $R$ ) as a function of tube length. (b) The variation of failure load as a function of tube length. (c) to (f) The normalized void volume fraction contour of tubes of different length to see the failure (void) nucleation sites. (c) Tube length of $20 \mathrm{~mm}$. (d) Tube length of $32 \mathrm{~mm}$. (e) Tube length of $40 \mathrm{~mm}$. (f) The critical load for tube failure during plane strain deformation. The load is normalized so that it is the failure force per unit length of the tube. Note the normalization is only applicable for flattening with platens. 
nucleation at the edges of the two ends of ultrathin tubes (see Fig. 5c). For longer tubes, we see more uniform damage nucleation at the two ends of the tubes (Fig. 5d), which leads to better compressibility. When tubes are even longer, damage nucleation tends to concentrate in the center of the two ends (see Fig. 5e and f). The localized damage in the center results in lower compressibility of longer tubes. The extreme case is when the tube is infinitely long, where the compressibility converges to that of the critical value from plane strain conditions in the tube axis direction. Based on the simulations shown in Fig. 5, we find that for flattening test with platens, it is desired that the tube length is about 1.2 $R$. Hence we could abstract reliable length-independent compressibility including both the failure flattening displacement and the corresponding load per unit length.

From all flattening simulations shown so far, we see that the loaddisplacement exhibit several characteristic stages: The initial elastic region, the following elastic-plastic region [16], and the region after failure initiation. Correspondingly, it is of interest to understand the three critical parameters associated with the characteristic deformation stages: The stiffness of the structure during the elastic response of the tube, the transitional point when plastic deformation starts, and the critical point when failure occurs. We first consider the elastic part during the flattening tests. Based on Castigliano's theorem and the structure symmetry [21] of the circular tube, we obtained the stiffness $K$ as

$K=\frac{F}{\delta}=\frac{4 \pi G E A^{2} M}{G R_{0}^{2} A^{2}\left(\pi^{2}-8\right)+\left[\left(16-\pi^{2}\right) G R_{0} A+k \pi^{2} R_{0} A E\right] M-8 G M^{2}}$

where $F$ is the applied compressive force and $\delta$ the resultant displacement, and $E, G$, and $k$, in turn, the elastic modulus, the elastic shear modulus, and shape parameter $k$. For the rectangular cross-section, we followed the reference [18] and adopted $\mathrm{k}=6 / 5$; and $R_{0}=R-t / 2$ is the radius of curvature of the bent lever centroidal axis, with $R$ the outer radius of tubes and $t$ thickness, and the static moment $M$ of the cross section for the neutral axis is defined as

$M=A\left(R-\frac{t}{2}-\frac{t}{\ln \frac{R}{R-t}}\right)$

for $A$ being the cross-sectional area of the bent lever. In Table 2 we list the stiffness of tubes with different thickness from FEM simulations and theoretical analysis.

In order to predict the transitional point when plastic deformation starts in the load-displacement curve from a flattening test, we took the following assumptions:

1. The thickness of the tube and the length of the neutral axis are unchanged before and after the flattening test. That is, the initial neutral line in arc $\widehat{A B}$ shown in Fig. 6a will remain the same after flattening (see Fig. 6b).

2. For the initially curved beam, the neutral and centroidal axis are not coincident [22]. The distance from the centroidal axis to the neutral axis, as illustrated by Fig. 6c, is given as

$e=R-\frac{t}{2}-\frac{t}{\ln \frac{R}{R-t}}$

Table 2

Comparison of the stiffness of tubes with different thickness from FEM simulations and theoretical analysis (Eqn. (4)).

\begin{tabular}{llll}
\hline Dimensionless thickness & $K(\mathrm{kN} / \mathrm{mm}, \mathrm{siml})$. & $K(\mathrm{kN} / \mathrm{mm}$, theo. $)$ & Difference \\
\hline 0.05 & 1.28 & 1.24 & $-3.23 \%$ \\
0.10 & 10.49 & 10.74 & $2.33 \%$ \\
0.15 & 38.26 & 39.15 & $2.27 \%$ \\
0.20 & 93.39 & 100.28 & $6.87 \%$ \\
\hline
\end{tabular}

Hence Eqn. (5) could be simplified as $M=A e$.

3. In addition, we assumed a uniform tensile stress distribution at any cross-section of the arc $\widehat{A B}$ before the plastic stage.

Thereafter, we could use the maximum elastic strain to predict the critical displacement of elastic stage $L_{c}$ in response to the emergence of plastic deformation. Geometrically, if we assumed that the deformed shape can be well represented by Fig. $6 \mathrm{~b}$ when plastic deformation embarks, $L_{c}$ can be expressed as

$L_{c}=2 R-H_{c}$

where

$H_{c}=\frac{\left(2 Q R-2 R^{2}\right)\left(1+\varepsilon_{e l}^{\max }\right)}{Q-R\left(1+\varepsilon_{e l}^{\max }\right)}$ with $Q=\frac{t}{\ln \frac{R}{R-t}}$

here $\varepsilon_{e l}^{\max }$ is the strain corresponding to the elastic limit of the tube material. From the stress-strain curve in Fig. 1b, we had $\varepsilon_{e l}^{\max }=\frac{\sigma_{y}}{E}=2.675 \times 10^{-3}$, for $\sigma_{y}$ the yield stress. From the load-displacement curves from FEM simulations, we might also define the point for the initiation of plasticity by the illustration shown in Fig. 6d, where point ' $\mathrm{A}$ ' is determined as the critical point. We compared the results of the critical displacement of elastic stage $L_{c}$ of samples with different thickness from FEM simulations and theoretical predictions. The results are listed in Table 3.

In Fig. 7, we show the predictability of Eqns. (4) and (7). The comparisons of the stiffness see in Fig. $7 \mathrm{a}$ and the critical displacement of the elastic stage shown in Fig. 7b suggest that the analyses are in good agreement with FEM results. We continue to show the predictability of the failure point. According to the stress and deformation characteristics of the flattening specimen [23], the failure displacement $L_{f}$, after being taken the offset of the neutral axis $e$ into account, is given by $L_{f}=2 R-H_{f}$, where $H_{f}$ is the failure ultra-distance between two platens and is written as

$H_{f}=\frac{(1+\alpha)(t+2 e) R}{\alpha R+\frac{t}{2}+e}$

where $\alpha$ is a material-dependent coefficient. For tubes made of alloy steels [24] $\alpha=0.09$. Eqn. (9) neglects the influence from the length of the flattened specimen. Hence it is by the best applicable to sufficiently long tubes under flattening. In Table 4 we show the results of failure displacement of tubes of the same outer radius of $200 \mathrm{~mm}$ but different thickness from FEM simulations and theoretical predictions. The corresponding plot is shown in Fig. 8. Simulation results for both plane strain and plane stress boundary conditions are presented. The agreement between theoretical analyses using Eqn. (9) and those from FEM simulations is reasonably well.

\section{Flattening test by indenters}

In the previous section, we presented results on flattening with platens. Technically, flattening with indenters is also broadly employed in engineering practice. We now consider the influence of tube geometries when indenters are used for flattening. We consider an indenter with geometries shown in Fig. 9a. The projected simulation setup, by using the isometric view, is given in Fig. 9b. Load-displacement curves from flattening tubes of different thicknesses using both platens and indenters are presented in Fig. 9c. The results indicate that for tubes with intermediate lengths, there is no perceivable difference between the two types of load-displacement curves from indenters and platens, respectively. However, the difference tends to grow when tube length increases. That is more clearly seen by examining the dependence of failure behavior on tube length. As shown in Fig. 10a, for short and intermediate tubes, the dependence of failure displacement on tube length from flattening with indenters is similar to that seen from 
(a)

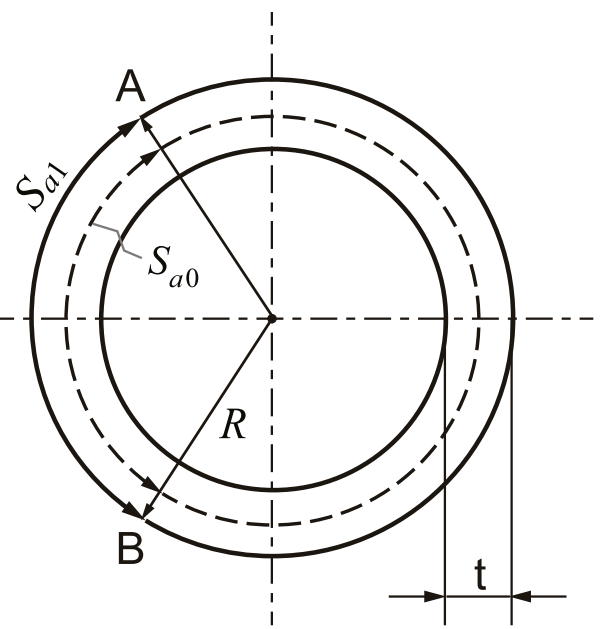

(c)

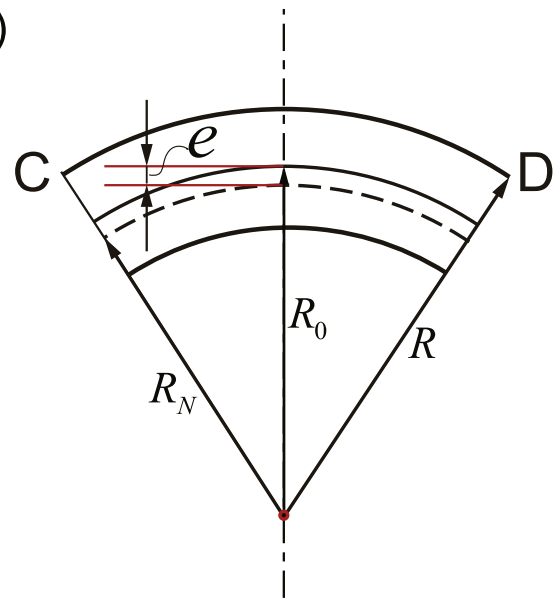

(b)

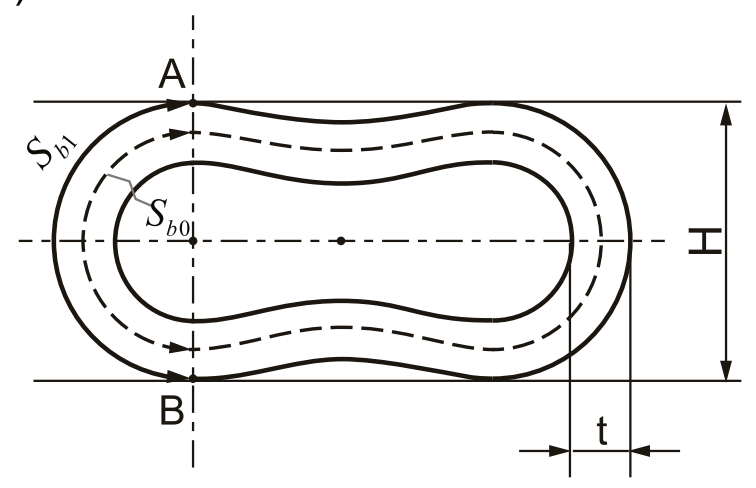

(d)

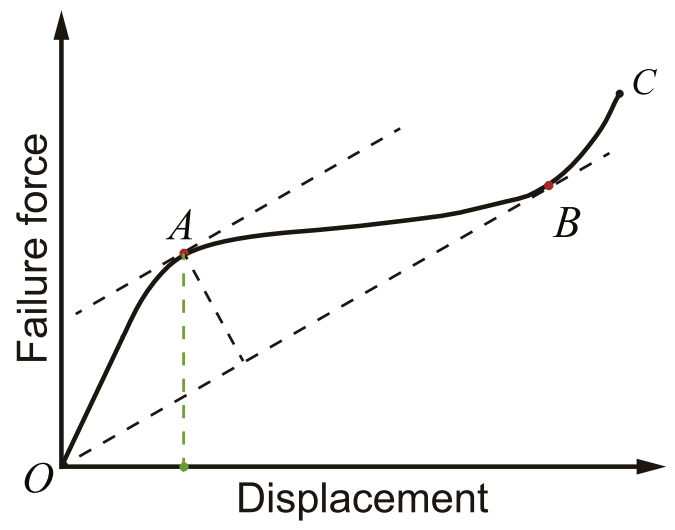

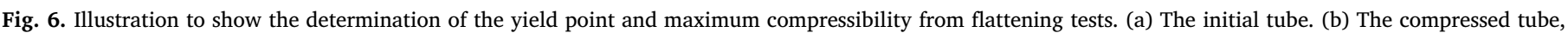

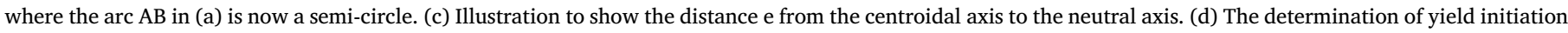
(point ' $A$ ') from the load-displacement curve from a flattening test.

Table 3

Comparison of the critical displacement of elastic stage with different tube thickness from FEM simulations and theoretical predictions.

\begin{tabular}{llll}
\hline Dimensionless thickness & $L_{c}(\mathrm{~mm}, \mathrm{siml})$. & $L_{c}(\mathrm{~mm}$, theo.) & Difference \\
\hline 0.05 & 35.32 & 37.40 & $5.56 \%$ \\
0.10 & 19.49 & 18.96 & $-2.80 \%$ \\
0.15 & 13.22 & 12.39 & $-6.70 \%$ \\
0.20 & 9.57 & 9.01 & $-6.22 \%$ \\
\hline
\end{tabular}

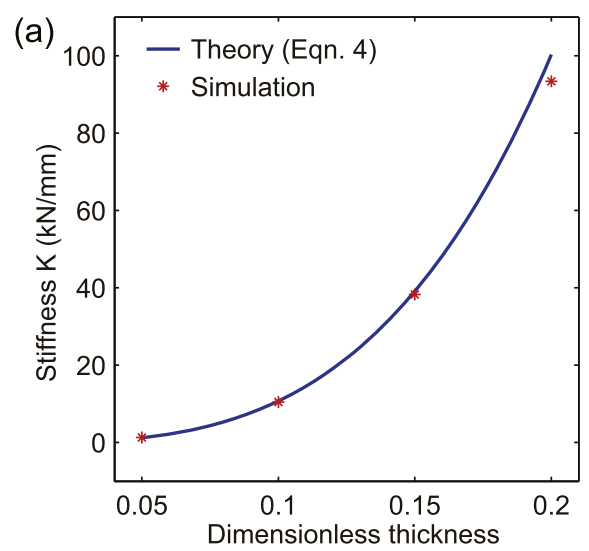

flattening with platens. It first increases and then decreases, in response to the transition of failure initiation sites from tube edges to the middle part (Fig. 5). Further increase in tube length would lead to better compressibility of the tube. As the tube becomes sufficiently long, the failure displacement for tubes under indenting converges to the value from the simulation with an infinitely long tube. From our simulation results, it seems that a tube length on the order of at least 4 times of the tube outer radius is good to obtain a length-independent compressible displacement. The failure load increases monotonically with tube

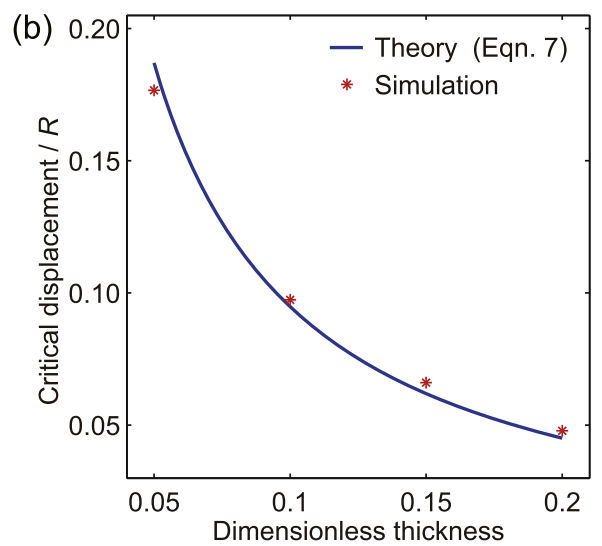

Fig. 7. The comparison of stiffness and the critical displacement of tubes from finite-element simulations and theoretical analysis. Tube thickness is normalized by tube outer radius $R$. (a) The stiffness as a function of tube thickness. (b) The critical displacement (divided by $R$ ) as a function of tube thickness. 
Table 4

Comparison of the failure displacement of tubes of different thickness from FEM simulations and theoretical predictions.

\begin{tabular}{lllll}
\hline $\begin{array}{l}\text { Dimensionless } \\
\text { thickness }\end{array}$ & Boundary & $\begin{array}{l}L_{f}(\mathrm{~mm}, \\
\text { siml.) }\end{array}$ & $\begin{array}{l}L_{f}(\mathrm{~mm}, \\
\text { theo. })\end{array}$ & Difference \\
\hline \multirow{2}{*}{0.05} & Plane strain & 314.28 & 304.58 & $-3.18 \%$ \\
& Plane stress & 329.75 & 304.58 & $-8.26 \%$ \\
0.10 & Plane strain & 247.63 & 242.54 & $-2.10 \%$ \\
& Plane stress & 253.13 & 242.54 & $-4.37 \%$ \\
0.15 & Plane strain & 206.74 & 198.93 & $-3.93 \%$ \\
0.20 & Plane stress & 200.25 & 198.93 & $-0.66 \%$ \\
& Plane strain & 175.87 & 166.56 & $-5.59 \%$ \\
& Plane stress & 172.55 & 166.56 & $-3.60 \%$ \\
\hline
\end{tabular}

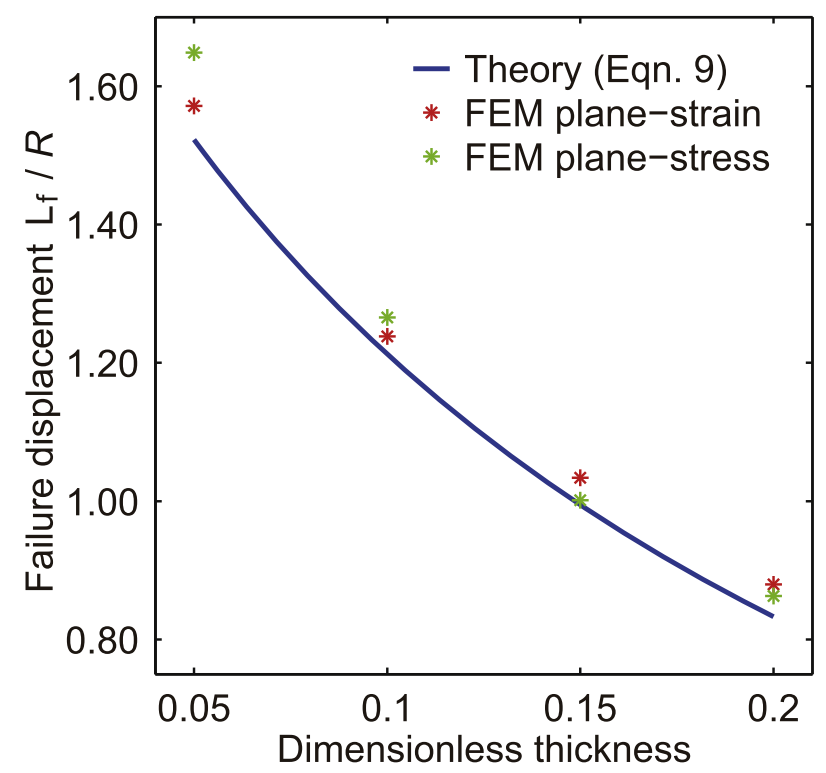

Fig. 8. The comparison of failure displacement (divided by tube outer radius $R$ ) of tubes of different thickness (normalized by $R$ ) from finite-element simulations (plain-strain and place-stress conditions) and theoretical analysis. length, but exhibits significant distinction at different tube length, as seen from FEM results shown in Fig. 10b. Till intermediate tube length, the failure load exhibits a degressive growth rate with tube length, and then a progressive increase in the failure load is observed for longer tubes. When the tube becomes even longer, the load now growth rate decreases again and gradually converges to the value when the tube length is infinite.

The deformation mechanisms accounting for the variation of both the failure displacement and failure load with tube length are explored. In Fig. 11, we show the deformed patterns of the cross-section (view from the lateral side of tubes) of the same thickness $(20 \mathrm{~mm})$. We considered four representative tubes under indenter flattening, three tubes with open ends and of length $200 \mathrm{~mm}, 800 \mathrm{~mm}, 2000 \mathrm{~mm}$, respectively, as well as an end-closed tube of length $2000 \mathrm{~mm}$. Fig. 11a shows the load-displacement curves of the four kinds of tubes. We show deformation patterns at different snapshots keyed in the load-displacement curve in Fig. 11a. We first show the deformation of the tube with length $200 \mathrm{~mm}$, as seen in Fig. 11b. Here the tube deforms as if under flattening with platens. All regions along the tube axis deforms uniformly. It hence explains the variation of failure displacement and failure load seen in Fig. 10 for short to intermediate tubes. Within this tube length region, the deformation of tubes under indenters resembles that under platens (see Fig. 5). Hence the first transition of the load in Fig. 10a, the initial increase and then decrease of the failure load as tube length increases is due to the transition of failure sites - from the edges in short tubes to the middle part in intermediate tubes. With further tube length increase, we see that the shape change along the tube axis is not uniform now, as seen in Fig. 11c for the tube of $800 \mathrm{~mm}$ in length. The non-uniform deformation is imposed by the constraint from the tube axis, which leads to the increase of the failure load after it reaches the minimum, as seen in Fig. 10a. After reaching the second peak and the boundary condition approaches the infinitely long tube case, the constraints at the two ends of the tube would reduce the compressibility slightly. When the tube length is sufficiently long, as for the case shown in Fig. 11d for the tube length of $2000 \mathrm{~mm}$, the deformation patterns themselves are converged, in consistent with the convergence in both the failure displacement and failure load seen in Fig. 10. For comparison, we also show in Fig. 11e the deformation patterns of an end-closed tube of the same thickness and length as in Fig. 11d. The deformation in this case may represent the deformation pattern of infinitely long tubes under indenting.

In Fig. 12, we present the void nucleation and growth sites in

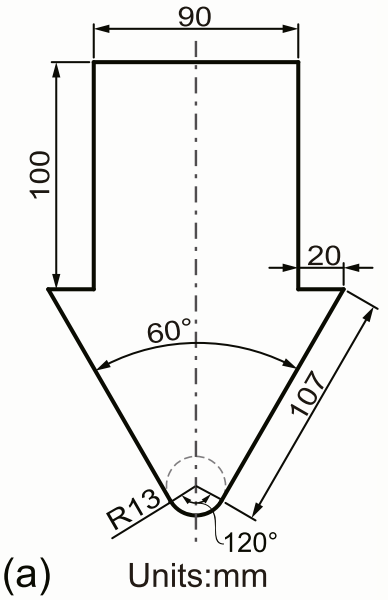

(a)

Units:mm
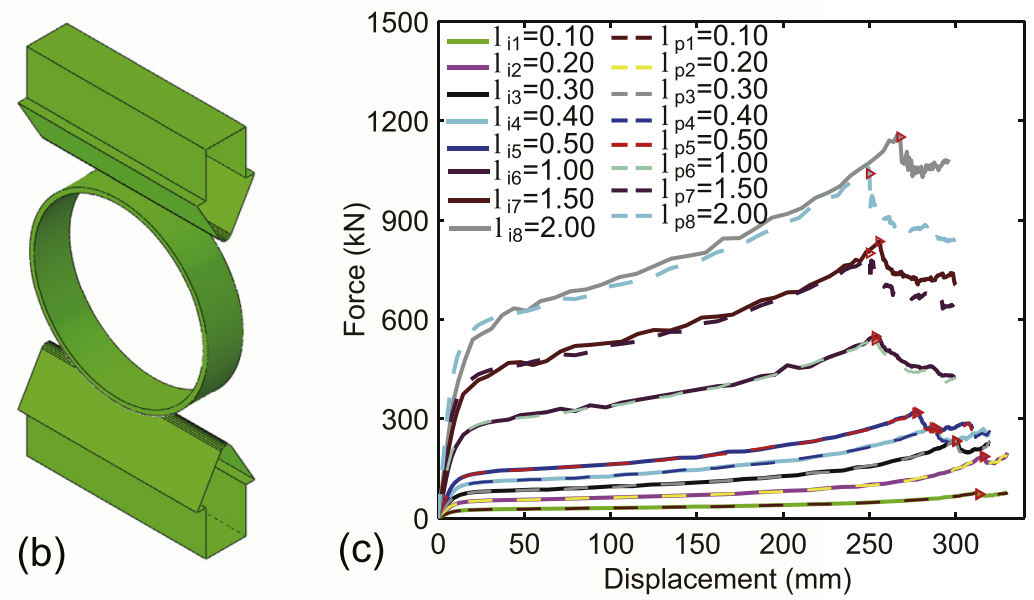

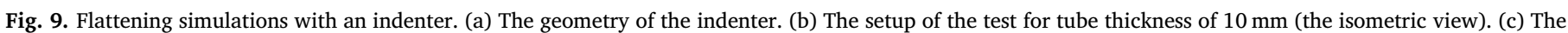

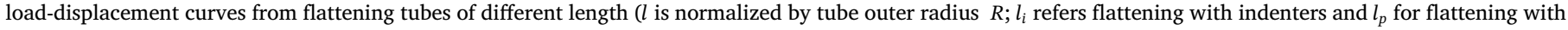
platens) from our finite-element simulation. For comparison, we also show load-displacement responses from flattening tubes with platens. 

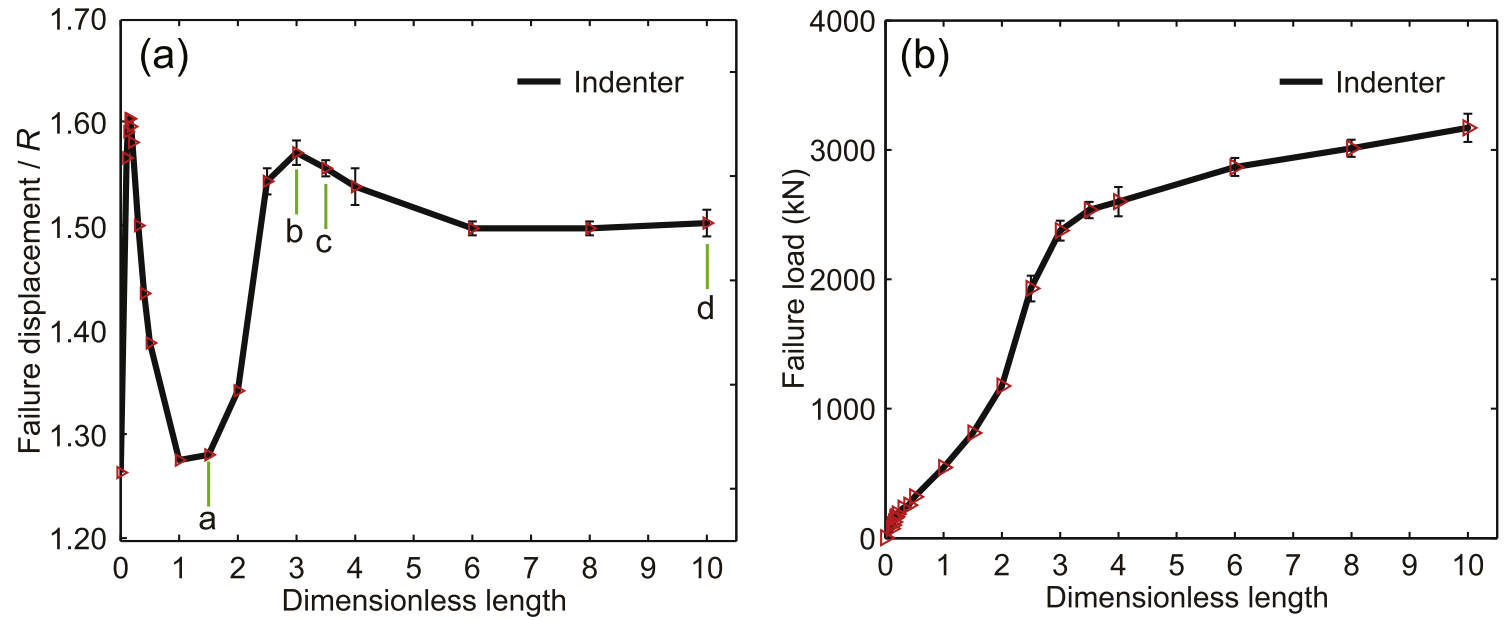

Fig. 10. The mechanical behavior in tubes of different length (normalized by tube outer radius $R$ ) but of the same thickness during flattening with indenters. (a) The variation of failure displacement (divided by $R$ ) as a function of tube length. (b) The variation of failure load as a function of tube length.

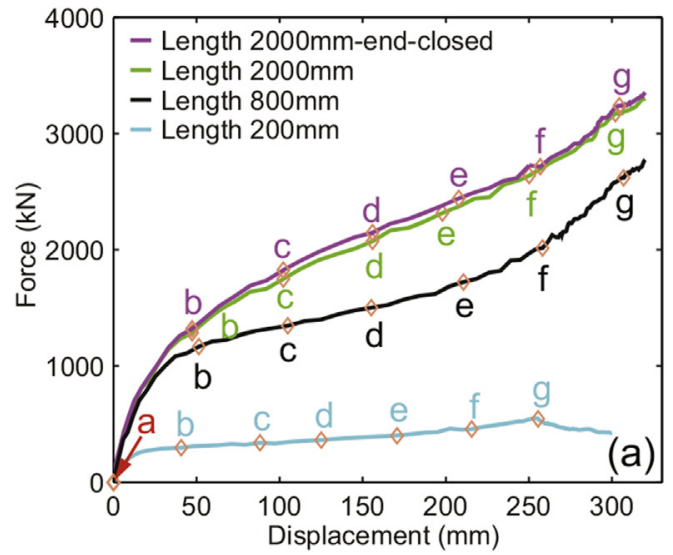

(b)

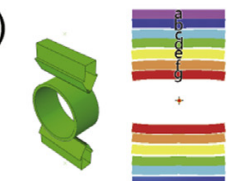

(c)

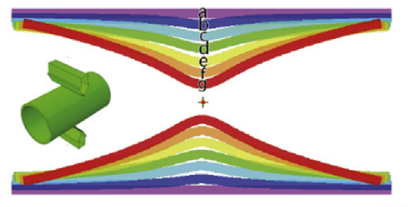

(d)

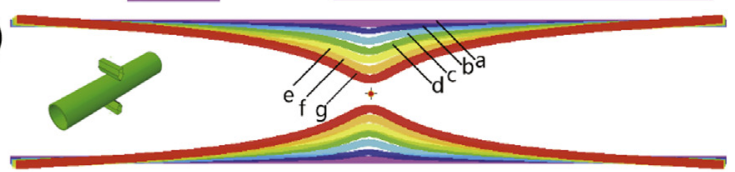

(e)

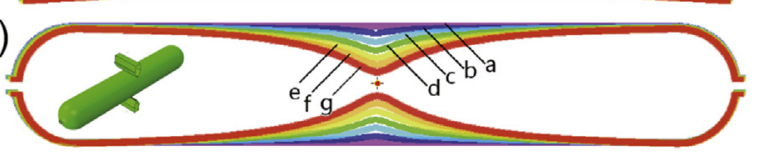

Fig. 11. Deformed patterns of the cross-section (view along the lateral side of tubes) of the same thickness (20 mm) and tube outer radius (200 mm). (a) The forcedisplacement curve of four kinds of tubes. Patterns at different displacement keyed in (a) will be shown. (b) A tube of length $200 \mathrm{~mm}$. (c) A tube of length $800 \mathrm{~mm}$. (d) A tube of length $2000 \mathrm{~mm}$. (e) An end-closed tube of length $2000 \mathrm{~mm}$.

(a)

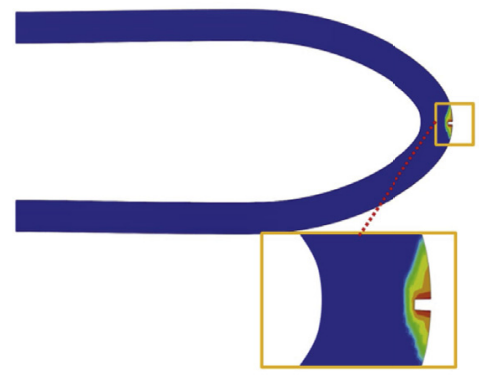

(c)

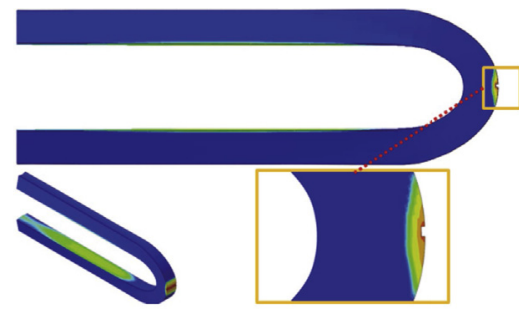

(b)

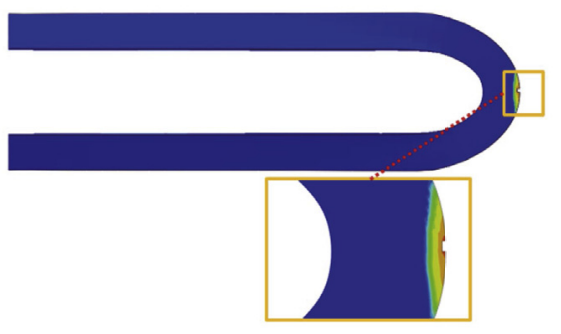

(d)

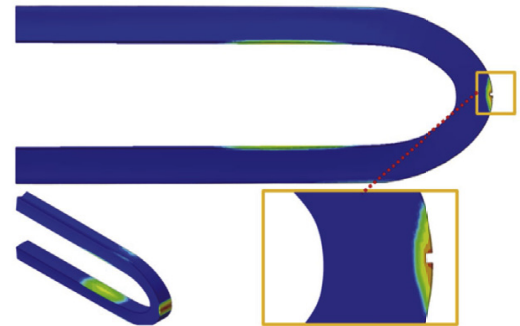

Fig. 12. Deformed patterns of the cross-section (perpendicular to the tube axis) of the same thickness $(20 \mathrm{~mm})$ and tube outer radius $(200 \mathrm{~mm})$. (a) to (d) Normalized void volume fraction contours at the failure displacement for tubes of different lengths keyed in Fig. 10a. (a) Tube length of $300 \mathrm{~mm}$. (b) $600 \mathrm{~mm}$. (c) $700 \mathrm{~mm}$. (d) $2000 \mathrm{~mm}$. 
flattening tests with indenters from the cross-section under the indenters (perpendicular to the tube axis). Fig. 12a-d shows normalized void volume fraction contours at the failure displacement for tubes of different lengths keyed in Fig. 10a. As explained, the first transition of compressibility in Fig. 10a is due to the transition of failure sites - from the edges in short tubes to the middle part in intermediate tubes. However, the shape change along the tube axis, which results in nonuniform displacement seen in Fig. 11, also promotes damage in the middle part of the cross-section. Fig. $12 \mathrm{c}$ and $\mathrm{d}$ for tubes longer than $700 \mathrm{~mm}$. The non-uniform displacement along the tube axis and the distributed damage promote the compressibility of the tubes under indenting, in consistent with the critical compressibility curve for tubes of different length shown in Fig. 10a.

\section{Conclusions and discussions}

With growing concerns about the security of structure like pipes, tubes and liquid tanks (cylinders), it is desired to give a thorough investigation about existing flattening tests. In particular, as non-standard experiments are still broadly used to evaluate the quality of tube materials, the validity and the accurate interpretation of those experimental results are crucial from both safety and economic considerations. Using the Gurson-Tvergaard-Needleman damage model [9-13] and finite element simulations to capture the flattening of a tube made of typical pipe materials, we reveal how the tube thickness, radius, and length would affect the displacement where tube failure initiates. More importantly, we build a connection between the load-displacement behavior of tubes under flattening with either platens or indenters and their deformation patterns. There is only one transitional behavior of the failure load (per unit length) for tubes under platen flattening: Failure initiation occurs at the two edges of short tubes, but shifts to the center in intermediate tubes. Such transition gives rise to an increase in the failure displacement and then decreases, and at the end, it converges to the limit case when the tube is infinitely long. For tubes under indenters, we see two transitions. The first transition of the failure load, similar to that seen in tubes under platen flattening, is due to the transition of failure sites - from the edges in short tubes to the middle part in intermediate tubes. The second transition originates from the geometrical feature of an indenter, it gives rise to uniform flattening along the tube axis when tubes are short, but non-uniform flattening emerges in intermediate and long tubes under indenting. Such nonuniform deformation, as imposed by the constraint from the tube axis, leads to the second increase of the failure load, and then both the failure load and the failure displacement converge to a value when the tube length becomes infinite. Those simulation results are in consistent with deformation patterns seen from different perspectives. Based on the simulations, we suggest that for flattening with platens, the tube length should be about $1.2 R$; for flattening with indenters, the tube length should be longer than $4 R$, in order to obtain equivalent results for flattening a whole gas tank or long tubes. We also supply theoretically analysis which could be applied to capture the mechanical characteristics in a typical load-displacement curve for tubes under platen flattening. The analysis shown here could be employed to characterize the mechanical properties of materials for pipes, tubes and tanks, and they could also be applied to guide the engineering design of such structures.

\section{Acknowledgments}

The authors acknowledge support from the National Natural Science Foundation of China (NSFC) (Grant no. 11425211), the Strategic Priority Research Program of the Chinese Academy of Sciences (XDB22020200), National Key R\&D Program of China (2017YFB1201302-10A) and Basic Scientific Research Foundation of Beijing Jiaotong University (Grant no. M14JB00130).

\section{Appendix A. Supplementary data}

Supplementary data to this article can be found online at https:// doi.org/10.1016/j.ijpvp.2019.01.007.

\section{References}

[1] L.B. Yan, N. Chouw, K. Jayaraman, Lateral crushing of empty and polyurethanefoam filled natural flax fabric reinforced epoxy composite tubes, Compos. B Eng. 63 (2014) 15-26.

[2] N.C. Tang, Plastic-deformation analysis in tube bending, Int. J. Pres. Ves. Pip. 77 (2000) 751-759.

[3] K.A. Babakri, Improvements in flattening test performance in high frequency induction welded steel pipe mill, J. Mater. Process. Technol. 210 (2010) 2171-2177.

[4] D. Munz, Cross-sectional flattening of pipes subjected to bending, Int. J. Pres. Ves. Pip. 10 (6) (1982) 421-429.

[5] M. Zohrabi, A. Niknejad, S. Ziaee, A novel method for enhancing energy absorption capability by thin-walled sections during the flattening process, Thin-Walled Struct. 97 (2015) 140-153.

[6] D. Mackenzie, J.T. Boyle, A simple pipe bend element for piping flexibility analysis, Int. J. Pres. Ves. Pip. 51 (1) (1992) 85-106.

[7] J. Rouzegar, H. Assaee, A. Niknejad, S.A. Elahi, Geometrical discontinuities effects on lateral crushing and energy absorption of tubular structures, Mater. Des. 65 (2015) 343-359.

[8] ISO/FDIS 8492: 2013(E), Metallic Materials-Tube-Flattening Test, (2013).

[9] A.L. Gurson, Continuum theory of ductile rupture by void nucleation and growth: path i-yield function and flow rules for porous ductile media, ASME Transaction, J Eng. Mater. Technol. 99 (1977) 2-17.

[10] V. Tvergaard, Influence of voids on shear band instabilities under plane strain conditions, Int. J. Fract. 17 (1981) 389-407.

[11] V. Tvergaard, On localization in ductile materials containing spherical voids, Int. J. Fract. 18 (1982) 237-252.

[12] V. Tvergaard, A. Needleman, Analysis of the cup-cone fracture in a round tensile bar, Acta Metall. 32 (1984) 157-169.

[13] C.C. Chu, A. Needleman, Void nucleation effects in biaxially stretched sheets, J. Eng. Mater. Technol. 102 (1980) 249-256.

[14] H. Li, M.W. Fu, J. Lu, H. Yang, Ductile fracture: experiments and computations, Int. J. Plast. 27 (2011) 147-180.

[15] K. Nahshon, Z. Xue, A modified Gurson model and its application to punch-out experiments, Eng. Fract. Mech. 76 (2009) 997-1009.

[16] D.J. Celentano, J.L. Chaboche, Experimental and numerical characterization of damage evolution in steels, Int. J. Plast. 23 (2007) 1739-1762.

[17] Y. Wang, G. Yang, W. Wang, X. Wang, Q. Li, Y. Wei, Optimal stress and deformation partition in gradient materials for better strength and tensile ductility: a numerical investigation, Sci. Rep. 7 (1) (2017) 10954.

[18] Abaqus FEA Edition 2007, D.S.S.D. Systèmes, 2007.

[19] D. Steglich, W. Brocks, Micromechanical modeling of the behavior of ductile materials including particles, Comput. Mater. Sci. 9 (1997) 7-17.

[20] D.Z. Sun, B. Voss, W. Schmitt, Numerical prediction of ductile fracture resistance behaviour based on micromechanical models, J. Appl. Physiol. 61 (1986) 1173-1179.

[21] J.M. Gere, S.P. Timoshenko, Mechanics of Materials, Second SI Edition, Van Nostrand Reinhold, New York, 1984.

[22] R.G. Budynas, Advanced Strength and Applied Stress Analysis, Second Edition, Mcgraw-Hill, New York, 1999.

[23] ASTM A370-2010, Standard Test Methods and Definitions for Mechanical Testing of Steel Products, (2010).

[24] P. Liang, X. Li, C. Du, X. Chen, Stress corrosion cracking of X80 pipeline steel in simulated alkaline soil solution, Mater. Des. 30 (5) (2009) 1712-1717. 